\title{
New Strategy for Streptavidin Detection Using AuNRs/PAAm Hydrogel Composites
}

\author{
Jean H. de Oliveira, ${ }^{a}$ Johny P. Monteiro, ${ }^{b}$ Paulo H. M. Buzzetti, ${ }^{\oplus a}$ Bento P. Cabral Jr., ${ }^{a}$ \\ Eduardo Radovanovic ${ }^{\odot a}$ and Emerson M. Girotto ${ }^{\circledR} * a$ \\ ${ }^{a}$ Laboratório de Química de Materiais e Sensores, Departamento de Química, \\ Universidade Estadual de Maringá, Avenida Colombo, 5790, 87020-900 Maringá-PR, Brazil \\ ${ }^{b}$ Departamento de Química, Universidade Tecnológica Federal do Paraná (UTFPR), \\ Rua Marcílio Dias, 635, 86812-460 Apucarana-PR, Brazil
}

\begin{abstract}
Localized surface plasmon resonance (LSPR) is a collective oscillation of free electrons induced by the incident light in nanometric surfaces of conductive materials. Gold nanorods (AuNRs) have been studied for plasmonic devices due to their anisotropic properties. Herein, we report the construction of a specific biosensor using polyacrylamide (PAAm) as the gold nanorods support and test its efficiency with specific streptavidin interaction using biotinylated substrates. First, the hydrogels were synthesized by electropolymerization using cyclic voltammetry on ITO/glass substrates and swallowed with AuNRs. The AuNRs were characterized by visible absorption spectroscopy and transmission electron microscopy. The substrates were characterized by scanning electron microscopy and their plasmonic sensitivities were evaluated using sucrose solutions with different concentrations. The results showed a sensitivity of ca. $226.4 \mathrm{~nm} \mathrm{RIU}{ }^{-1}$ with linear correlation of 0.9974 . Furthermore, this composite presented reliable detection of the streptavidin biomolecule in the gold surface with good signal-to-noise ratio (SNR).
\end{abstract}

Keywords: gold nanorods, plasmonic biosensor, streptavidin, hydrogel

\section{Introduction}

The advances in sensors area are continuously encouraging the research for new materials. The localized surface plasmon resonance (LSPR) is a versatile phenomenon to sensors application; its mechanism is based on interactions between electromagnetic waves and metallic nanostructures that can be useful in optical devices for chemical and biological sensing. ${ }^{1,2}$ Gold is one of the most popular materials used on LSPR basedsensors due to its noble properties. Gold nanostructures are extremely sensitive to changes in the dielectric environment they are in. Due to the confinement effect of the electromagnetic radiation, these nanostructures are very sensitive to events close to the surface. One of these interactions is a shift in the LSPR absorption band as the refractive index (RI) changes or molecules bind on the gold surface. ${ }^{3}$ LSPR phenomenon depends on many factors such as geometry, composition and interaction between the particles. Associated with the plasmonic effects, several studies show different

*e-mail: emgirotto@uem.br interactions for the light-gold system based on the geometry, such as nanoshells, ${ }^{4}$ nanoholes, ${ }^{5}$ nanoprisms, ${ }^{6}$ and nanorods. ${ }^{7}$ Gold nanorods (AuNRs) present some attractive optical properties due to their anisotropic structure. These structures show two absorption bands in the electromagnetic spectrum: a maximum wavelength corresponding to transversal plasmonic oscillations, and other to longitudinal oscillations. ${ }^{8,9}$

Hydrogels are three-dimensional polymeric networks that swell in water without dissolution. Their properties play key roles in several technological applications, including absorbents, ${ }^{10}$ drug delivery systems, ${ }^{11}$ sensors, ${ }^{12}$ and other areas. ${ }^{13-15}$ Hydrophilic chains allow interactions between hydrogels and gold nanostructures, consisting on an efficient method to make biosensors since both materials are biocompatible and can interact with each other keeping the structure intact for new interactions. ${ }^{16}$ SPR using hydrogel films open new possibilities in sensors area leading to new types of detection using this combination, such as sensors for bovine serum albumin, ${ }^{17}$ fusion gene, ${ }^{18}$ and hydrogen peroxide. ${ }^{19}$

In this work, we prepared gold nanorods capable of anchoring onto the polyacrylamide (PAAm) hydrogel by 
electrostatic interactions with amine-terminated groups. The composite was prepared by immersion in AuNRs solution and its sensitivity was studied by absorption spectroscopy in the visible region and finally tested for biotin/streptavidin to characterize the sensor efficiency.

\section{Experimental}

\section{Chemicals}

Cetyltrimethylammonium bromide (CTAB), $\mathrm{HAuCl}_{4} \cdot 4 \mathrm{H}_{2} \mathrm{O}$, ascorbic acid (AA), acrylamide, cysteamine hydrochloride, biotin-sulfo-NHS (BNHS), $N, N^{\prime}$-methylenebisacrylamide (MBAA), silver nitrate, sodium borohydride, and streptavidin were purchased from Sigma-Aldrich (São Paulo, SP, Brazil). Zinc chloride was purchased from ECIBRA ${ }^{\circledR}$ (Santo Amaro, SP, Brazil). The ITO/glass slides were purchased from Adafruit ${ }^{\circledR}$ (New York, NY, USA). All chemicals, unless mentioned otherwise, were of analytical reagent grade and purchased from Anidrol (Diadema, SP, Brazil). Deionized water was used in solutions preparation.

\section{AuNRs synthesis}

Gold nanorods were prepared as described by Sau and Murphy ${ }^{20}$ using the well-established seed mediated method. Initially, the seed solution was prepared by adding $250 \mu \mathrm{L}$ of $\mathrm{HAuCl}_{4}\left(0.01 \mathrm{~mol} \mathrm{~L}^{-1}\right)$ in $7.5 \mathrm{~mL}$ of CTAB $\left(0.1 \mathrm{~mol} \mathrm{~L}^{-1}\right)$ and gently stirring at room temperature $(\mathrm{rt})$. Then, $0.6 \mathrm{~mL}$ of ice cooled $\mathrm{NaBH}_{4}\left(0.01 \mathrm{~mol} \mathrm{~L}^{-1}\right)$ was added, turning the solution into a bright brown color with gentle mixing. The solution was stored at $\mathrm{rt}$ for $2 \mathrm{~h}$ before use. The grown solution was firstly obtained by adding $4.75 \mathrm{~mL}$ of CTAB $\left(0.1 \mathrm{~mol} \mathrm{~L}^{-1}\right), 0.20 \mathrm{~mL}$ of $0.01 \mathrm{~mol} \mathrm{~L}^{-1}$ $\mathrm{HAuCl}_{4}$, and $30 \mu \mathrm{L}$ of $0.01 \mathrm{~mol} \mathrm{~L}^{-1} \mathrm{AgNO}_{3}$, sequentially, in a falcon tube, followed by gentle mixing by inversion. In this stage, the solution appeared bright brown-yellow. Then, $32 \mu \mathrm{L}$ of $0.10 \mathrm{~mol} \mathrm{~L}^{-1} \mathrm{AA}$ was added, turning the solution colorless. Finally, $10 \mu \mathrm{L}$ of the seed solution was added, and the reaction mixture was gently mixed for $10 \mathrm{~s}$ and left undisturbed for at least $3 \mathrm{~h}$. For the composite synthesis, the nanorods were centrifuged at $2000 \mathrm{rpm}$ for $10 \mathrm{~min}$ to remove the agglomerates, and next centrifuged at $10000 \mathrm{rpm}$ two times and re-suspended in water to remove the CTAB excess.

\section{Hydrogel synthesis}

The PAAm hydrogel was synthesized by electropolymerization using cyclic voltammetry with acrylamide, zinc chloride and MBAA. The electropolymerization experiment was reported in a previous study. ${ }^{17}$ The ITO/glass substrates were washed and sonicated with deionized water and acetone for $5 \mathrm{~min}$ and dried with nitrogen flow. The cyclic voltammetries (5 cycles) were done from -1.5 to $0.2 \mathrm{~V}$, holding in $-1.5 \mathrm{~V}$ for $20 \mathrm{~s}$ before starting the next cycle. A platinum wire was used as counter electrode, $\mathrm{Ag} \mid \mathrm{AgCl}$ as reference electrode and the ITO substrate as working electrode. After polymerization, the substrates were immersed in $\mathrm{HCl}$ solution $\left(0.01 \mathrm{~mol} \mathrm{~L}^{-1}\right)$ for $24 \mathrm{~h}$ to remove the excess of metallic zinc. Finally, the substrates were shrunken in acetone and immersed in water for 2 min to remove the excess of acid.

\section{PAAm/AuNRs composite preparation}

First, the substrates were immersed in the nanorods solution for $12 \mathrm{~h}$ to obtain a homogeneous coating. The composites were washed with deionized water for the next step. To ensure the performance of the sensor, a plasmonic sensitivity was measured using sucrose solutions with different RI (1.3328 to 1.4022), by inserting the substrates in the glucose solution for $15 \mathrm{~min}$ and making the spectra in deionized water.

\section{Biosensitivity evaluation}

The substrates were immersed in a cysteamine solution $\left(50 \mu \mathrm{mol} \mathrm{L}{ }^{-1}\right)$ for $12 \mathrm{~h}$, followed by the immersion in an aqueous solution of BNHS $\left(2 \mathrm{mg} \mathrm{mL}^{-1}\right)$ for $4 \mathrm{~h}$, and finally in the streptavidin solution $\left(2.5 \mathrm{mg} \mathrm{mL}^{-1}\right)$ for $4 \mathrm{~h}$ to complete the sensor, evaluating each step by UV-Vis spectroscopy.

\section{Instrumentation}

The AuNRs were visualized by transmission electron microscopy (TEM, JEOL JEM 1400) at an accelerating voltage of $120 \mathrm{keV}$ and counted using the software Image Pro-Plus ${ }^{\circledR}$ v. $7.0 .{ }^{21} \mathrm{~A}$ close examination of the composite formation was also evaluated by TEM. Ocean Optics USB 2000 spectrometer was used to obtain the spectra of AuNRs solution, and also in the sensitivity and biosensitivity tests. All the UV-Vis spectra were obtained using the experimental setup shown in Figure 1. To evaluate the morphology of the PAAm substrates it was used the scanning electron microscopy (SEM, Shimadzu SEM-S550) and their porous count was measured using the software Image Pro-Plus ${ }^{\circledR}$ v. 7.0. ${ }^{21}$ 


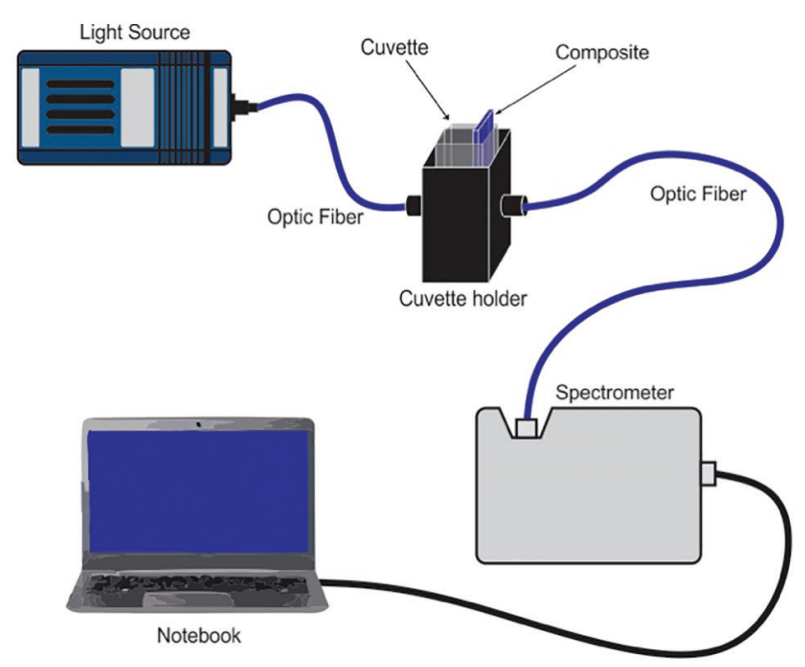

Figure 1. Illustrative scheme of the experimental setup used for measuring the sensitivity and biosensitivity of the composites.

\section{Results and Discussion}

Figure 2a presents the UV-Vis absorption spectra of the purified AuNRs in solution (right) that shows a small band at $\lambda_{\max }=518.3$ and a large one at $\lambda_{\max }=755.1 \mathrm{~nm}$. The shorter wavelength band is related to the transverse plasmon mode. The shoulder in this band is related to unexpected particles. On the other hand, the more red-shifted band is associated with the longitudinal plasmon mode. Compared to spherical shaped gold nanoparticles, the AuNRs offer some different characteristics in plasmon oscillations. Gold nanorods show two surface plasmon modes: the longitudinal LSPR mode is associated with the electron oscillations along the length axis, while the transverse LSPR mode is excited by light polarized along the transverse direction of the AuNRs. ${ }^{22}$ The morphological analysis of the AuNRs was observed by the TEM image (Figure 2b), which presented homogeneous rods with some non-grown particles. The average diameter and length obtained by the image were $(14.1 \pm 1.9) \mathrm{nm}$ and $(45.4 \pm 5.5) \mathrm{nm}$, respectively, as observed in the size distribution histogram (in-graph).

Figure 3 presents the cyclic voltammogram of the PAAm supported in the ITO/glass surface. It indicates the zinc reduction, starting in ca. $-1.1 \mathrm{~V}$, during cathodic scanning, and also the acrylamide polymerization, which occurred via complex formation in the oxidation step. This behavior has already been described by Collins and Thomas. ${ }^{23}$ It is possible to observe that the signal decreased with the electropolymerization cycles, which indicates the reduction of electroactive area in the synthesis tending to a limit of the layer thickness. ${ }^{24}$

The micrograph of the PAAm (Figure 4a) showed a porous and homogeneous structure with an average pore size of $(3.92 \pm 1.10) \mu \mathrm{m}$. Metallic zinc was used to form this uniform honeycomb structure, being dissolved after in the acid bath releasing a pure hydrogel matrix. The thickness of the hydrogel was ca. $65 \mu \mathrm{m}$ (Figure $4 \mathrm{~b}$ ), which can be useful to absorb a large quantity of molecules and nanorods to finish the biosensor.

Figure 5 shows the TEM images of the AuNRs immobilization in the hydrogel matrix. As seen in the images, the nanorods were encapsulated within the hydrogel matrix (shaded region of the TEM image). In addition, the AuNRs seem to present a side interaction that kept them aligned in parallel (even with some spherical particles appearing).

The sensitivity evaluation was performed using sucrose solutions with different RIs. Figure 6a shows the plasmonic response of the ITO/PAAm/AuNRs sensor.
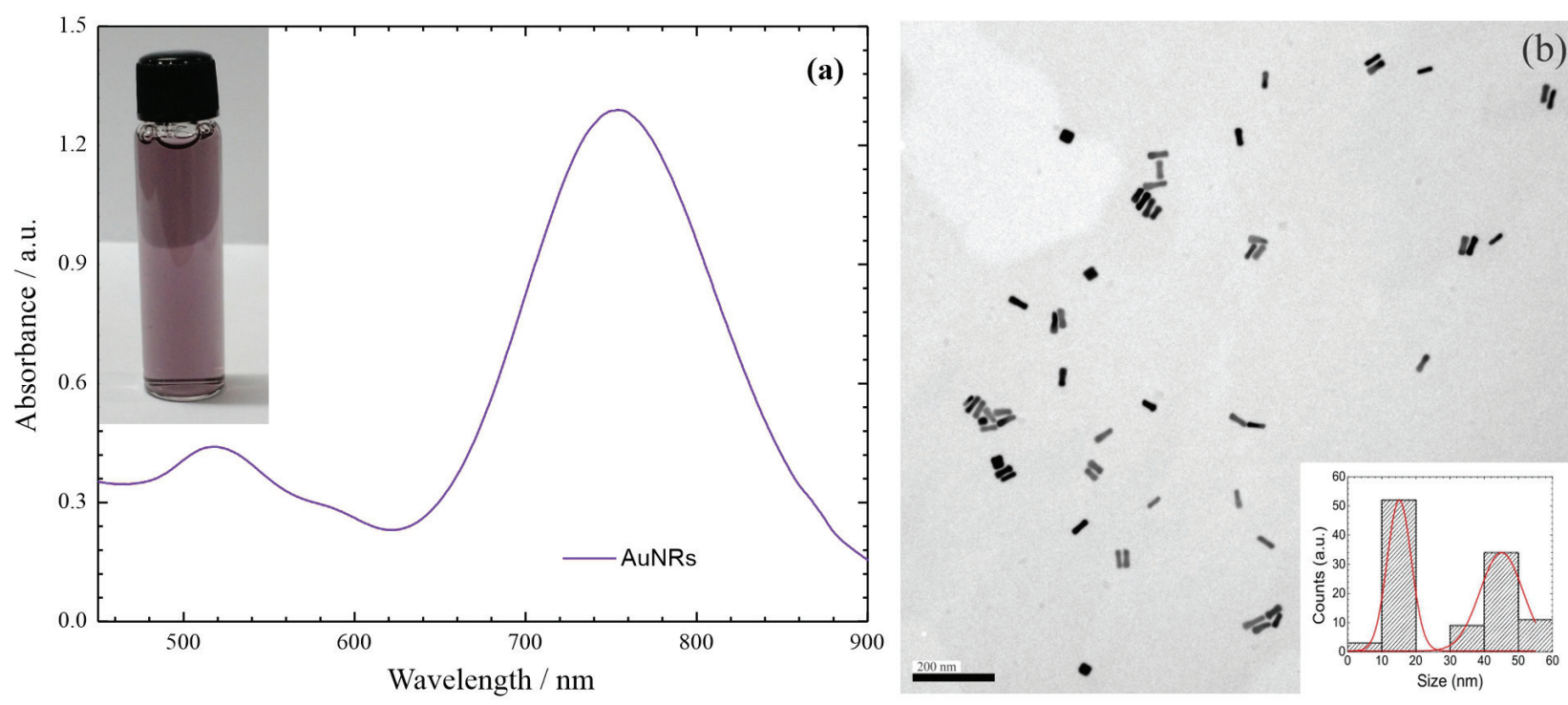

Figure 2. UV-Vis spectra for diluted gold nanorods solution (a) and TEM image after the centrifugation with size distribution histogram (b). 


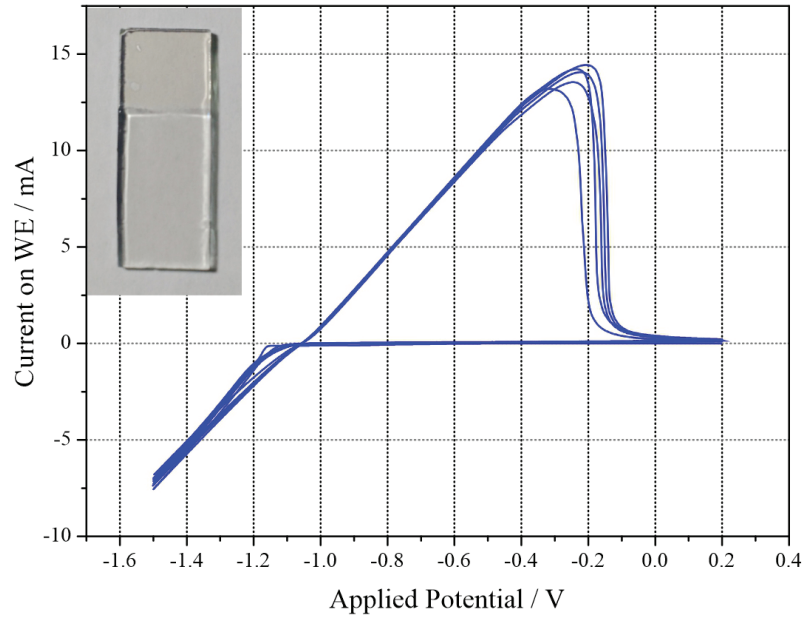

Figure 3. Cyclic voltammogram of the electropolymerized PAAm hydrogel with a photograph after the zinc removal.
This redshift corresponds to the different localized surface plasmon in the gold structure. The plasmonic right peak position redshifts as the RI of the solution increases. ${ }^{25}$ The CTAB on the AuNRs surface can facilitate the functionalization or interaction of the AuNRs tips (occurred from the face $\{001\}$ exposed) because the surfactant bilayer causes an obstruction along the rods, and this indicates the changes in the longitudinal plasmonic peak to the redshift. ${ }^{26}$

Figure $6 \mathrm{~b}$ shows the sensitivity evaluation for the ITO/PAAm/AuNRs composite, which presented a value of ca. $226.4 \mathrm{~nm} \mathrm{RIU}{ }^{-1}$, close to the sensitivity found by Mayer et al..$^{27}$ for the gold nanorods in a previous report. The coefficient of regression of 0.9974 presents a coherent regression line to the data obtained. Other relevant

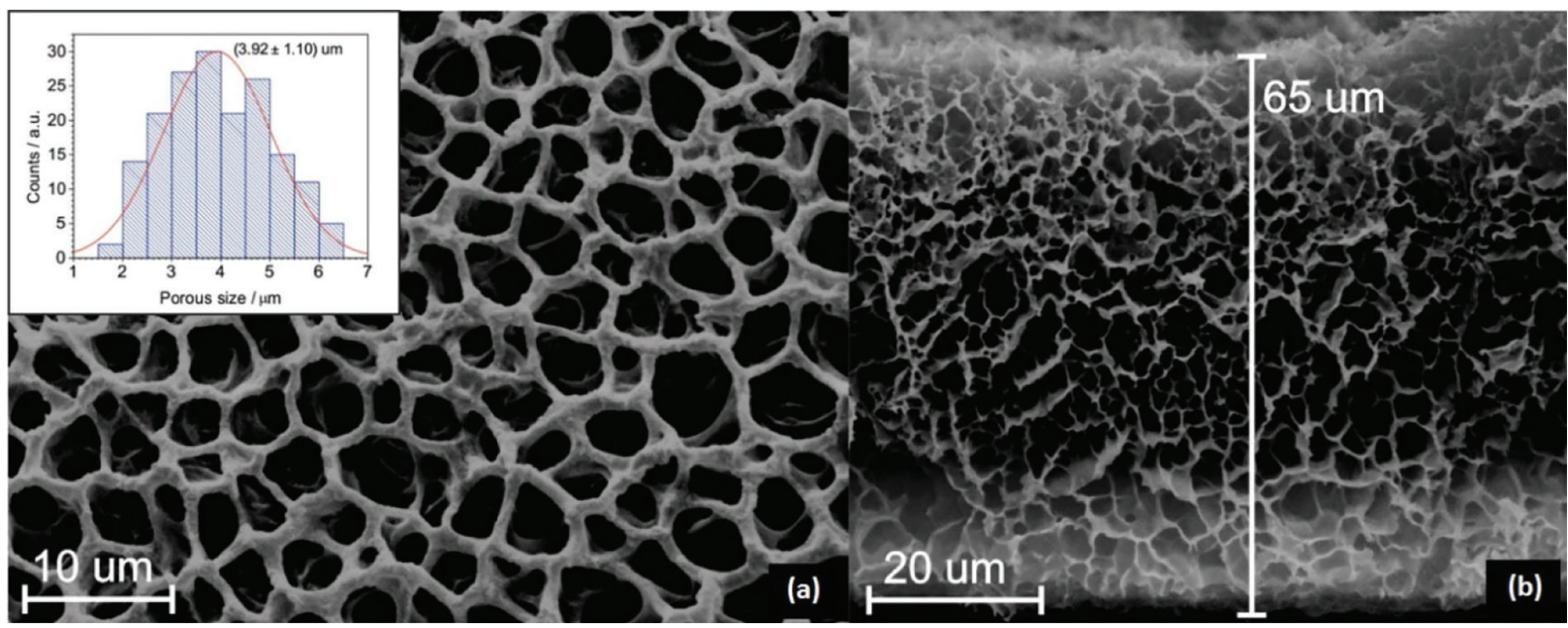

Figure 4. Scanning electron micrographs (SEM) of the PAAm hydrogel without the nanorods modification.

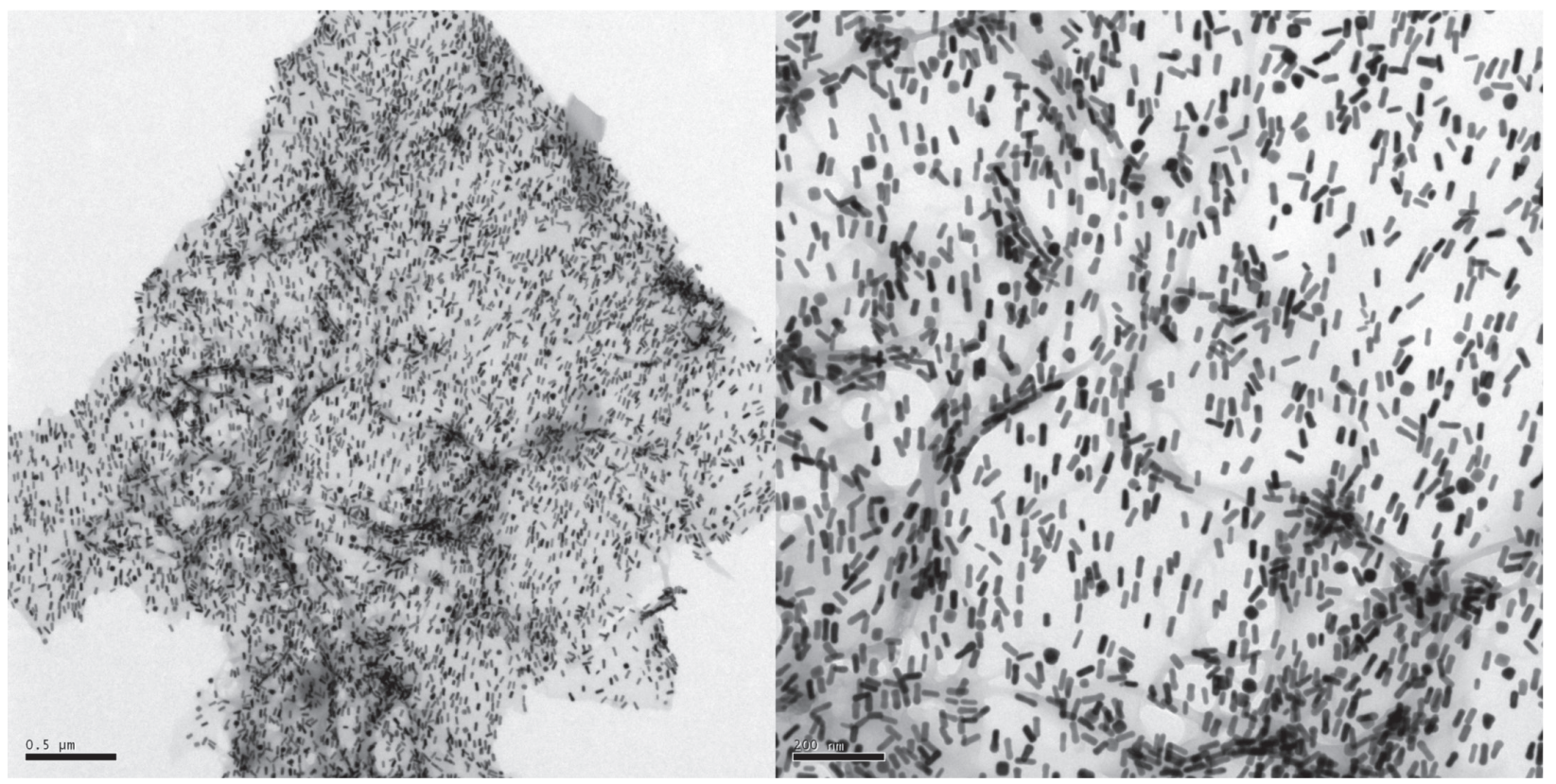

Figure 5. TEM images of the AuNRs anchored in the hydrogel matrix with close magnification. 

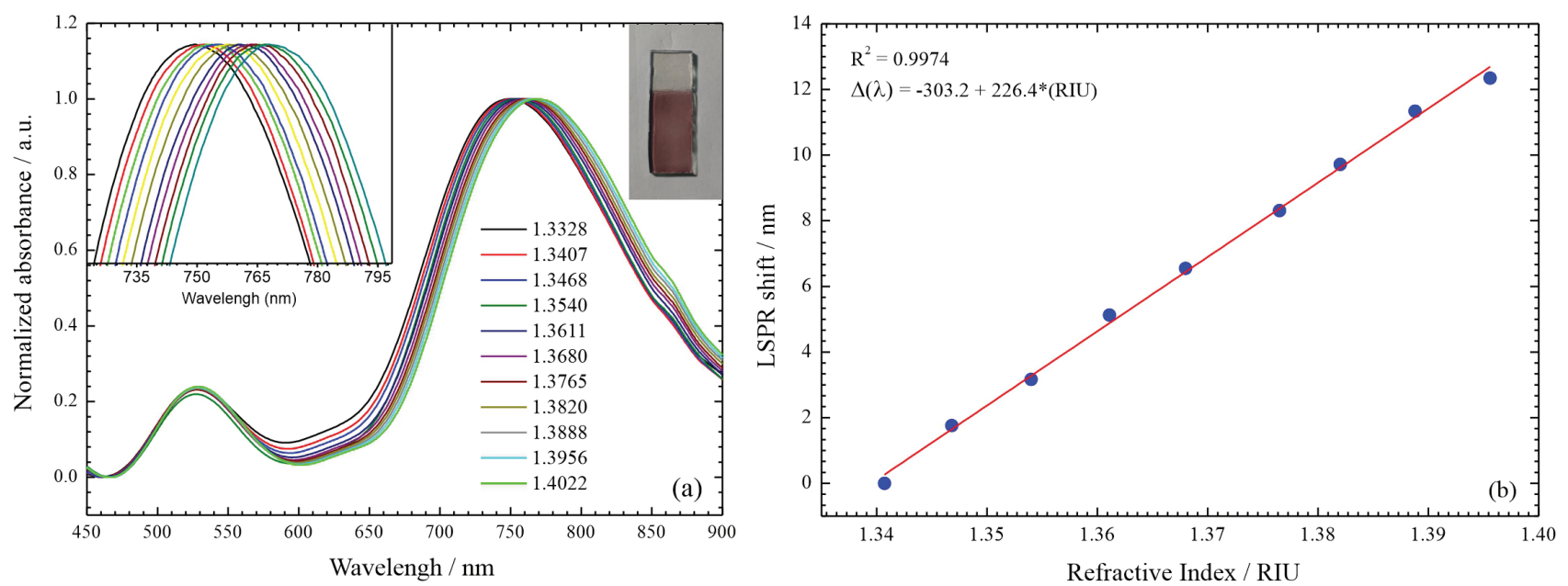

Figure 6. (a) Visible absorption spectra of ITO/PAAm/AuNRs composite in sucrose solutions with close approximation of the longitudinal $\lambda_{\max }$ and (b) sensitivity graph for the ITO/PAAm/AuNRs composite.

parameter is the resolution $(\mathrm{R})$, which corresponds to the small change in the refractive index that produces a detectable shift in the sensor output. ${ }^{28}$ Considering that the precision of the system was $0.1 \mathrm{~nm}$, and the sensitivity obtained, the resulting $\mathrm{R}$ value from the experimental setup was $\mathrm{R}=4.4 \times 10^{-4} \mathrm{RIU}$, indicating the accuracy of our biosensor for LSPR. In a previous work, our research group obtained a similar detection system for bovine serum albumin (BSA) based on graphene and gold nanoparticles with a resolution of $2.2 \times 10^{-4}$ RIU. ${ }^{29}$ For R values lower than $10^{-4}$ RIU, the system can detect extremely small changes in the RI, which allows the detection of low concentrations of entities bonded at the AuNR surface. ${ }^{30}$

The biosensitivity evaluation was performed first with the immobilization of cysteamine (Cys) onto the AuNRs surface present in the hydrogel. Cys molecules interact strongly with the gold surface by -SH groups, leaving the $-\mathrm{NH}_{2}$ group free for further modifications. ${ }^{31,32}$ Based on this purpose, the second step was anchor the biotin in the Cys surface, which led to a biotinylated surface proper for streptavidin detection. The N-hydroxysuccinimide group at the biotin molecule can be easily removed from the reaction with the exposed amine group of the Cys/AuNRs system..$^{33}$ When biotin is exposed on the substrate surface it can interact specifically with streptavidin molecules in a cross-linked mechanism due to their binding affinity $\left(\mathrm{K}_{\mathrm{a}}\right.$ ca. $\left.10^{-3} \mathrm{~L} \mathrm{~mol}^{-1}\right)$, being one of the strongest receptorbinder interaction. ${ }^{34,35}$

Visible absorption spectroscopy in different solutions was used to test the biosensor efficiency. According to Figure 7, it was observed a redshift of the plasmonic band after each functionalization. The cysteamine presented a shift of ca. $7 \mathrm{~nm}$, higher than that observed in plasmonic systems based on gold nanostructures. ${ }^{36}$ Unlike cysteamine, biotin exhibited a redshift of $2 \mathrm{~nm}$, and the streptavidin redshifted almost $9 \mathrm{~nm}$.

The displacement of ca. $9 \mathrm{~nm}$ after the insertion of streptavidin can be explained by two reasons. First, the modification of the metal surface in systems involving gold nanostructures with similar sensitivity showed similar displacement as described in the literature. ${ }^{37}$ Second, streptavidin is a large molecule that significantly affects the displacement value, since there are molecules not anchored in the metal surface, but adsorbed in the gel matrix, inducing a larger redshift. ${ }^{17}$

To test whether the substrate can be used as a biosensor it is important to verify the signal-to-noise ratio (SNR) that leads to a useful platform to detect streptavidin in this type of sensor. The plasmonic band shift of streptavidin can be visualized considering the error bar in Figure 7, which indicated a substantial SNR.

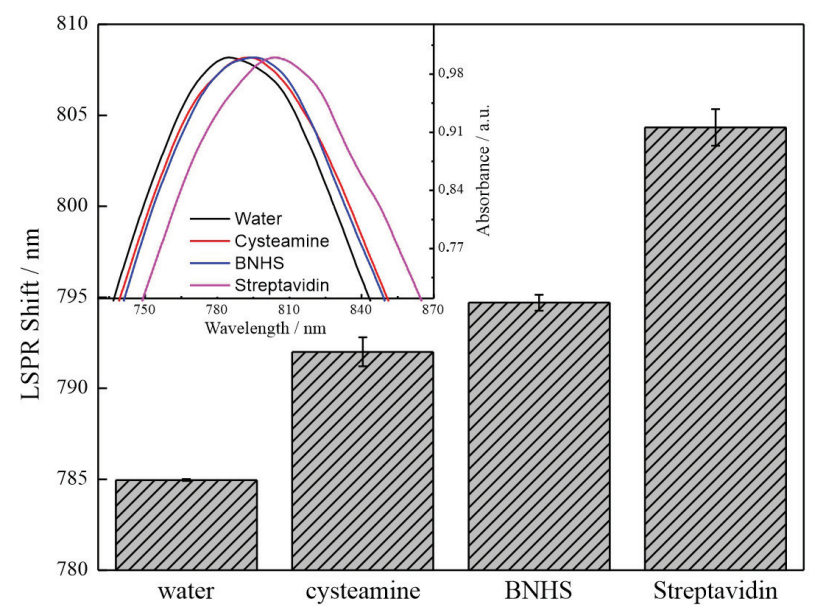

Figure 7. Redshift observed with biofunctionalization of the substrate with cysteamine, BNHS and streptavidin, with a close view of the longitudinal $\lambda_{\max }$ of the absorption spectrum (in-graph). 
The detection of streptavidin is a preliminary study in order to prepare a label-free biosensing method. Cabral Jr. et al..$^{5}$ prepared a cost-effective substrate for streptavidin detection achieving a sensitivity of ca. $380 \mathrm{~nm} \mathrm{RIU}{ }^{-1}$ using nanoholed systems. Another platform published by Focsan et al. ${ }^{38}$ reached a detection limit of $5 \mathrm{nM}$ of streptavidin using plasmonic gold nanostructures in assembled layers. Thus, we were able to generate a new sensing platform using films of AuNRs anchored in a PAAm hydrogel matrix to detect streptavidin in low concentrations $\left(2.5 \mathrm{mg} \mathrm{mL}^{-1}\right)$.

\section{Conclusions}

In this work, were produced polyacrylamide hydrogel/ AuNRs composites, which successfully detected low concentrations of biotin-streptavidin. The hydrogel presented a uniform coating on the ITO surface with high porous distribution, allowing further modifications. The ITO/PAAm/AuNRs biosensor showed good sensitivity in sucrose solutions with different RI and good response to specific modification using cross-linked test biotinstreptavidin. A redshift of ca. $9 \mathrm{~nm}$ indicated the adsorption of the streptavidin in the gold surface, allowing its detection.

\section{Acknowledgments}

The authors acknowledge the Brazilian agencies CAPES and CNPQ for financial support and the Complex of Research Support Centers (COMCAP) for the microscopical analyses.

\section{Author Contributions}

Jean $\mathrm{H}$. de Oliveira was responsible for the investigation, methodology, software, validation, writing the original draft, writing-review and editing; Johny P. Monteiro for the investigation, methodology and writing-review and editing; Bento P. C. Junior for the investigation, data curation and formal analysis; Paulo H. M. Buzzetti for data curation, methodology and conceptualization; Eduardo Radovanovic for conceptualization, methodology, validation and formal analysis; Emerson M. Girotto for conceptualization, funding acquisition, project administration, writing original draft and writing-review and editing.

\section{References}

1. Thakur, A.; Qiu, G.; NG, S.-P.; Guan, J.; Yue, J.; Lee, Y.; Wu, C.-M. L.; Biosens. Bioelectron. 2017, 94, 400.
2. Fu, L.; Li, C.; Li, Y.; Chen, S.; Long, Y.; Zeng, R.; Sens. Actuators, B 2017, 240, 315.

3. Wang, S.; Sun, X.; Ding, M.; Peng, G.; Qi, Y.; Wang, Y.; Ren, J.; J. Phys. D: Appl. Phys. 2018, 51, 045101.

4. Song, J.; Yang, X.; Yang, Z.; Lin, L.; Liu, Y.; Zhou, Z.; Shen, Z.; Yu, G.; Dai, Y.; Jacobson, O.; Munasinghe, J.; Yung, B.; Teng, G.-J.; Chen, X.; ACS Nano 2017, 11, 6102.

5. Cabral Jr., B. P.; de Oliveira, J. H.; Buzzetti, P. H. M.; Fressatti, M. G. V.; Monteiro, J. P.; Amorin, L. H. C.; Urbano, A.; Radovanovic, E.; Girotto, E. M.; Mater. Lett. 2018, 227, 243.

6. Liu, W.; Tian, Y.; Zhang, Y.; Liu, K.; Zhao, S.; Zhang, J.; Su, Y.; Zhao, Y.; Tang, Y.; Sun, J.; Tian, W.; Song, L.; Teng, Z.; Wang, S.; Lu, G.; J. Mater. Chem. B 2018, 6, 3865.

7. Azab, M. M.; Cherif, R.; Finnie, A. L.; Abou El-Alamin, M. M.; Sultan, M. A.; Wark, A. W.; Analyst 2018, 143, 1635.

8. Wang, P.; Zhang, L.; Xia, Y.; Tong, L.; Xu, X.; Ying, Y.; Nano Lett. 2012, 12, 3145.

9. Han, X.; Fang, X.; Shi, A.; Wang, J.; Zhang, Y.; Anal. Biochem. 2013, 443, 117.

10. Yue, M.; Imai, K.; Yamashita, C.; Miura, Y.; Hoshino, Y.; Macromol. Chem. Phys. 2017, 218, 1600570.

11. Seeli, D. S.; Prabaharan, M.; Carbohydr. Polym. 2017, 158, 51.

12. Lei, Z.; Wang, Q.; Wu, P.; Mater. Horiz. 2017, 4, 694.

13. Yang, C.; Suo, Z.; Nat. Rev. Mater. 2018, 3, 125.

14. Silva, D.; Fernandes, A. C.; Nunes, T. G.; Colaço, R.; Serro, A. P.; Acta Biomater. 2015, 26, 184.

15. Martins, A. F.; Monteiro, J. P.; Bonafé, E. G.; Gerola, A. P.; Silva, C. T. P.; Girotto, E. M.; Rubira, A. F.; Muniz, E. C.; Chin. Chem. Lett. 2015, 26, 1129.

16. Tanaka, K.; Naka, K.; Miyoshi, E.; Narita, A.; Chujo, Y.; Polym. J. 2016, 48, 177.

17. Monteiro, J. P.; Predabon, S. M.; da Silva, C. T. P.; Radovanovic, E.; Girotto, E. M.; J. Appl. Polym. Sci. 2015, 132, 42449.

18. Guo, B.; Wen, B.; Cheng, W.; Zhou, X.; Duan, X.; Zhao, M.; Xia, Q.; Ding, S.; Biosens. Bioelectron. 2018, 112, 120.

19. Nguyen, C. T.; Kasi, R. M.; Chem. Commun. 2015, 51, 12174.

20. Sau, T. K.; Murphy, C. J.; Langmuir 2004, 20, 6414.

21. Media Cibernetics, Inc.; Image Pro Plus; Maryland, USA, 2010.

22. Huang, X.; El-Sayed, I. H.; Qian, W.; El-Sayed, M. A.; J. Am. Chem. Soc. 2006, 128, 2115.

23. Collins, G. L.; Thomas, N. W.; J. Polym. Sci. 1977, 15, 1819.

24. Karpenko, M. A.; Kolzunova, L. G.; Russ. J. Electrochem. 2011, 47, 1091

25. Mock, J. J.; Smith, D. R.; Schultz, S.; Nano Lett. 2003, 3, 485.

26. Caswell, K. K.; Wilson, J. N.; Bunz, U. H. F.; Murphy, C. J.; J. Am. Chem. Soc. 2003, 125, 13914.

27. Mayer, K. M.; Lee, S.; Liao, H.; Rostro, B. C.; Fuentes, A.; Scully, P. T.; Nehl, C. L.; Hafner, J. H.; ACS Nano 2008, $2,687$.

28. Homola, J.; Chem. Rev. 2008, 108, 462. 
29. da Silva, C. T. P.; Monteiro, J. P.; Radovanovic, E.; Girotto, E. M.; Sens. Actuators, B 2014, 191, 152.

30. Tian, L.; Chen, E.; Gandra, N.; Abbas, A.; Singamaneni, S.; Langmuir 2012, 28, 17435.

31. Tan, S. F.; Anand, U.; Mirsaidov, U.; ACS Nano 2017, 11, 1633.

32. Love, J. C.; Estroff, L. A.; Kriebel, J. K.; Nuzzo, R. G.; Whitesides, G. M.; Chem. Rev. 2005, 105, 1103.

33. Yam, C.-M.; Pradier, C.-M.; Salmain, M.; Marcus, P.; Jaouen, G.; J. Colloid Interface Sci. 2001, 235, 183.

34. DeChancie, J.; Houk, K. N.; J. Am. Chem. Soc. 2007, 129, 5419.
35. Pérez-Luna, V. H.; O’Brien, M. J.; Opperman, K. A.; Hampton, P. D.; López, G. P.; Klumb, L. A.; Stayton, P. S.; J. Am. Chem. Soc. 1999, 121, 6469.

36. Vogel, N.; Jung, M.; Bocchio, N. L.; Retsch, M.; Kreiter, M.; Köper, I.; Small 2010, 6, 104.

37. Hu, Y.; Song, Y.; Wang, Y.; Di, J.; Thin Solid Films 2011, 519, 6605.

38. Focsan, M.; Campu, A.; Craciun, A. M.; Potara, M.; Leordean, C.; Maniu, D.; Astilean, S.; Biosens. Bioelectron. 2016, 86, 728.

Submitted: July 10, 2019

Published online: March 3, 2020 